\title{
Relationship of Dielectric Property Change to Composite Material State Degradation
}

\author{
Rassel Raihan $_{1}$, Jon-Michael Adkins 1 , Jeffrey Baker 1, Fazle Rabbi $_{1}$, Kenneth Reifsnider ${ }_{1}{ }^{*}$
}

${ }_{1}$ University of South Carolina, Mechanical Engineering, 300 Main Street, Columbia, SC

$$
\text { 29208, USA }
$$

\begin{abstract}
:
Polymer matrix composites are widely used in many industries, i.e. aerospace, microelectronics, energy storage etc., because of their unique properties and performance. During their service life, changes of material state caused by deformation and damage accumulation under combined mechanical, thermal and electrical fields requires fundamental understanding to support design of those material systems. Heterogeneous material systems are inherently dielectric as determined by their complex morphology. Dielectric properties of such materials are altered by many factors, e.g., electrical and structural interactions of the particles, and the shape, orientation and distribution of the constituents of the material system. When damage occurs, new phases are created as microdefects, and grow progressively, interact, and accumulate. The dielectric properties of the composite system also change in a manner that uniquely reflects those details. In the present work we report a non-invasive, in-operando technique to study changes in dielectric properties during progressive damage accumulation in composite materials subjected to mechanical loading.
\end{abstract}

Keywords: A. Flexible Composites, A. Polymer-Matrix Composites, B. Matrix Cracking, B. Electrical Properties, C. Crack, D. Broadband Dielectric Spectroscopy (BbDS)

*Corresponding Author

E-mail address: reifsnider@cec.sc.edu

(C) 2014. This manuscript version is made available under the Elsevier user license

http://www.elsevier.com/open-access/userlicense/1.0/ 


\section{Introduction:}

Applications of heterogeneous fiber reinforced polymer matrix composite materials require an understanding of the relationships that exist between the functional characteristics of material systems and the long-term behavior of those materials under combined mechanical, thermal, and electrical fields. In general, the heterogeneity of composite materials has been introduced and engineered to achieve specific properties and functions. These material systems are designed in such a way that they can withstand distributed micro damage, e.g., matrix micro-cracking, or multiple fiber breaks, even in the same fiber. Single micro- defects do not individually alter the strength of the composite materials, but when these defects accumulate and interact with each other during the service life, significant changes in the engineering properties of the material system can occur. The taxonomy of these changes as a function of the constituents and loading histories is still incomplete, especially as they relate to the development of final fracture events.

Degradation of composite materials is generally initiated and evolves by microdamage development events, especially matrix microcracking and crack growth, delamination, fiber fracture, fiber-matrix debonding, and microbuckling [1]. Progressive failure in composite materials can be considered to be the statistical accumulation, and subsequent interaction between microdefects. During dynamic tensile loading, acceleration of the damage accumulation can be rate dependent, and can alter the ultimate strength, strain to failure, and energy dissipation capabilities [2]. David Hufner [3], Fig. 1, showed that the nonlinear stress-strain behavior of off-axis woven composite materials can be divided into four damage zones, as we will discuss below.

*Corresponding Author

E-mail address: reifsnider@cec.sc.edu 
There are many Non-destructive techniques used to detect the complex damage in composite materials. Ultrasonic Testing (UT) and Acoustic Emission (AE) techniques are widely used NDT methods for composite defect detection [4-7]. In ultrasonic NDE, elastic waves propagate through the sample. Flaws/damage cause disturbances in the wave field which can be detected typically by utilizing one the following measurements: time of flight (TOF; wave transit or delay), path length, frequency, phase angle, amplitude, acoustic impedance, and angle of wave deflection (reflection and refraction). Scanning Acoustic Microscopy (SAM) defect detection techniques are high resolution ultrasonic imaging methods that apply a range of frequencies from $30 \mathrm{MHZ}$ to $3 \mathrm{GHz}$. Figure 2.3 is the schematic of the SAM technique often used to detect delamination in composite laminates [8]. Both of these techniques have specific advantages and applicability; they also have some limitations such as the requirement of point to point inspection, limited penetration depth due to attenuation, limited interpretation due to a high variation of composite properties, low contrast due to high attenuation and scattering in composites, and slow methodology. Acoustic Emission (AE) is also a well-established NDE method [4], but due to the multiple reflections in composite materials, wave propagation speed dependence on direction, and attenuation, the "triangulating" of damage in composite materials using AE signals is difficult.

Most NDE techniques require sensors that contact the surface of the composites or are non-contact methods that must have a clear view of the sample surface. But in electrical methods, most of the time, the composite system itself is used as the sensor. Detecting damage using electrical resistivity is a well-known practice for damage detection in composite research [9-21]. Irving et al. [21] have studied electrical resistance changes for 
both static and fatigue testing of CFRP. Although electrical resistance methods have the potential to characterize the damage state during the service life of composite materials, they do not give quantitative information about the defects, orientation of the flaws, crack density, and local morphology, or specific information about damage modes.

Broadband dielectric spectroscopy is used to study the interaction of electromagnetic waves with matter, usually in the frequency range from $10^{-6}$ to $10^{12} \mathrm{~Hz}$. This dynamic range generally can provide information about the molecular and collective dipolar fluctuations, as well as about charge transport and polarization effects that occur at inner and outer boundaries in the form of different dielectric properties of the material under study. Fig 2. shows the effect of different charge displacement mechanisms on dielectric response and their corresponding effective frequency ranges. Hence, broadband dielectric spectroscopy can also be used as an effective tool to detect and interpret the damage state of heterogeneous material systems.

Electromagnetic phenomena can be described by four equations constructed by Maxwell [25].

$\nabla \cdot \vec{D}=\rho$

$\nabla \times \overrightarrow{\boldsymbol{H}}=\vec{\jmath}+\frac{\partial \overrightarrow{\boldsymbol{D}}}{\partial t}$

$\nabla \times \overrightarrow{\boldsymbol{E}}+\frac{\partial \overrightarrow{\boldsymbol{B}}}{\partial t}=0$

$\nabla \cdot \vec{B}=0$ 
Here $\overrightarrow{\boldsymbol{D}}$ is the dielectric displacement, $\rho$ is the charge density, $\overrightarrow{\boldsymbol{H}}$ the magnetic field, $\overrightarrow{\boldsymbol{E}}$ the electric field, $\overrightarrow{\boldsymbol{B}}$ is the magnetic induction, and $\vec{\jmath}$ is the ohmic current density. In addition to Maxwell's equations, the field must satisfy continuity equations based on the charge density $\rho$ and current density $\vec{\jmath}$ which can be expressed as follows

$$
\nabla \cdot \vec{\jmath}+\frac{\partial \rho}{\partial t}=0
$$

For linear materials the interrelation between the dielectric displacement $\overrightarrow{\boldsymbol{D}}$ and electric field $\overrightarrow{\boldsymbol{E}}$ can be expressed as

$$
\overrightarrow{\boldsymbol{D}}=\varepsilon_{0} \overrightarrow{\boldsymbol{E}}+\overrightarrow{\boldsymbol{P}}
$$

and polarization can be related to the charge density when no external source is present by the following equation

$$
\nabla \cdot \overrightarrow{\boldsymbol{P}}=-\rho
$$

For a linear relationship between the dielectric displacement $\overrightarrow{\boldsymbol{D}}$ and electric field $\overrightarrow{\boldsymbol{E}}$ the proportionality constant $\varepsilon$ can be used to express

$\overrightarrow{\boldsymbol{D}}=\varepsilon \varepsilon_{0} \overrightarrow{\boldsymbol{E}}$

Here the constant $\varepsilon$ is called the permittivity of the material which describes the dielectric behavior, normalized by $\varepsilon_{0}$, the dielectric constant of vacuum. When the Polarization $\overrightarrow{\boldsymbol{P}}$ is taken into consideration using (6) and (8) we obtain

$\overrightarrow{\boldsymbol{P}}=\chi \varepsilon_{0} \overrightarrow{\boldsymbol{E}} \equiv \varepsilon_{0}(\varepsilon-1) \overrightarrow{\boldsymbol{E}}$ 
Here $\chi$ is the polarization coefficient known as the dielectric susceptibility.

The dependence of the complex dielectric function $\varepsilon^{*}(\omega)$ on the angular frequency $\omega$ of the applied electric field and temperature can generally be attributed to the following

1) Microscopic fluctuations of molecular dipoles.

2) Propagation of mobile charge carriers by translational diffusion of electrons, holes or ions.

3) The separation of charges at interfaces which gives rise to an additional polarization. The latter can take place at inner dielectric boundary layers (Maxwell/Wagner/Sillars-polarization) on a mesoscopic scale [26].

Each of the above mentioned processes have specific features in the frequency and temperature dependence of the real and imaginary part of the complex dielectric function.

Fazzino, et al. studied polymer based composites and showed that when they develop micro cracking due to mechanical loading, their dielectric response changed dramatically and definitively [23]. They used end-loaded bending, which caused surface initiation of damage, for fatigue loading of woven glass fiber reinforced polymer composites. Glass fibers are well known for their hydrophilic behavior [26] and when glass fiber reinforced polymer composites are exposed to water or ionic solutions, they penetrate by diffusion into the materials. Fazzino's samples were soaked in $5 \mathrm{M} \mathrm{NaCl}$ solution which diffused into the micro cracks and penetrated through the surface towards the center of the sample. The response was very sensitive to the local morphology (including the topological connectivity of the dielectric dispersed phase, e.g., the micro cracks in this case). In the lower frequency region, samples became more conductive as damage accumulated, as clearly shown in Figs. 3 and 4 . A general method of using broadband dielectric 
spectroscopy $(\mathrm{BbDS})$ to detect and interpret the damage state in composite materials was introduced in 2009 by Reifsnider et al. [ 24]

In this paper we introduce a unique in-situ dielectric property measurement technique that has the ability to identify damage modes in a composite material system using interpretations of the changes in the dielectric response during the progression of damage induced by quasi-static mechanical loading.

\section{Materials and Methods:}

\subsection{Material:}

The composite material NP130 was chosen for these experiments, and was provided by Norplex-Micarta, a manufacturer of high performance thermoset composite laminates. The thin laminated composites used for tensile testing were made with a plain weave, Eglass fabric combined with a halogenated epoxy resin matrix. The manufacturers estimated $55 \%$ volume fraction of fiber in that material. The samples were composed of five plies, wherein each ply consisted of a single layer of fabric with two principal fiber directions of 0 and 90 degrees, with the wrap direction defined as the 0 degree orientation. The total thickness of the samples was nominally $1 \mathrm{~mm}$. The samples were cut off-axis, at 45 degrees relative to the principal axis.

\subsection{In-situ Tensile Testing Configuration:}

To perform the uniaxial tensile extension of coupon specimens of the woven glass fiber reinforced epoxy composites, MTS Landmark ${ }^{\mathrm{TM}}$ software was used to drive the quasi-static test while coupled with a Novocontrol ${ }^{\mathrm{TM}}$ unit (which has high phase accuracy and wide impedance range) by a special set-up where strain was measured with an extensometer attached to the sample. A parallel plate capacitor arrangement was also 
attached to the sample and was connected to the analyzer of the Novocontrol ${ }^{\mathrm{TM}}$ unit. To form the parallel plate capacitor arrangement, two copper electrodes were attached permanently on two polycarbonate base plates and positioned in such a way that both copper electrodes faced each other. Samples were placed in-between the two plates and constant electrode pressure on the surface of the sample was maintained with a springloaded clamp throughout the test. The analyzer measured the complex dielectric, conductivity, and impedance properties of the test materials as a function of frequency of the applied electric field, with very high precision. The test system was especially optimized for dielectric materials with high impedance and low loss factors over a broad frequency range, but can also accurately measure the response of highly conductive materials with low impedance. Figure 5 shows the experimental setup used to measure the dielectric properties. The in-situ dielectric measurement at $10 \mathrm{~Hz}$ was used to interpret data from the AC signal applied by specially designed contact plates fixed on opposing sides of the thickness of the specimens.

Multiple specimens were loaded in tension and held at different load levels during the in-situ testing, and an edge replication technique was applied to quantify the damage state of the material.

\subsection{Principles of Dielectric Measurement in the NOVOCONTRL ${ }^{\text {TM}}$ :}

The sample material was mounted in a sample cell between two electrodes forming a sample capacitor as shown in Fig 6 . A voltage $U_{0}$ with a fixed frequency, $\omega / 2 \pi$, was applied to the sample capacitor. $\mathrm{U}_{0}$ caused a current $\mathrm{I}_{0}$ at the same frequency in the sample. In addition, there was generally a phase shift between current and voltage described by the phase angle $\varphi$ as shown in Fig 7.

*Corresponding Author

E-mail address: reifsnider@cec.sc.edu 
The ratio between $U_{0}$ and $I_{0}$ and the phase angle $\varphi$ are determined by the sample material electric properties (permittivity and conductivity) and by the sample geometry. So the appropriate relations in complex notation can be expressed as

$$
\begin{aligned}
& U(t)=U_{0} \cos (\omega t)=\operatorname{Re}\left(U^{*} \exp (i \omega t)\right) \\
& I(t)=I_{0} \cos (\omega t+\varphi)=\operatorname{Re}\left(I^{*} \exp (i \omega t)\right)
\end{aligned}
$$

with $U^{*}=U_{0}$ and $I^{*}=I^{\prime}+i I^{\prime \prime}$ where $I_{0}=\sqrt{I^{2}+I^{\prime \prime}}$ and $\tan (\varphi)=\frac{I^{\prime \prime}}{I^{\prime}}$.

For a sample with linear electromagnetic response, the measured impedance of the sample capacitor

$$
Z^{*}=Z^{\prime}+i Z^{\prime \prime}=\frac{U^{*}}{I^{*}}
$$

and the imaginary permittivity can be calculated by

$\varepsilon^{*}(\omega)=\varepsilon^{\prime}-i \varepsilon^{\prime \prime}=\frac{-i}{\omega Z^{*}(\omega)} \cdot \frac{1}{C_{0}}$

Here $C_{0}$ is the capacity of the empty sample capacitor.

The specific conductivity is related to the dielectric function by

$$
\sigma^{*}(\omega)=\sigma^{\prime}-i \sigma^{\prime \prime}=i 2 \pi f \varepsilon_{0}\left(\varepsilon^{*}-1\right)
$$

\section{Results And Discussion:}

\subsection{Dielectric Property Analysis}

Uniaxial tensile tests of off-axis coupon samples were performed. Dielectric property changes were measured during the total range of loading until the samples underwent material fracture. As suggested by Hufner [3], we divided the stress-strain curve of the off-axis woven composite samples into four zones, Fig. 8, based on the changes in dielectric property and damage progression. 
Fig. 9 shows the observed behavior of the real part of the permittivity / electrical compliance at a single frequency $(10 \mathrm{~Hz})$ which was recorded in-situ during the loading period. There is an observed decrease in the real part of the permittivity during the final period when fiber fractures lead to specimen failure.

Changes in the imaginary part of the permittivity during the loading are shown in Fig. 10. During the early stages of loading there was a significant increase in this property of the material. . For these off axis samples, abrupt changes occurred in the loading region in which distributed matrix damage occurred.

In heterogeneous materials systems, interfacial polarization plays a vital role in the response at lower frequencies of the applied electrical field [30]. When constituents with different conductivity are used in a composite material, interfacial charge accumulates and polarization occurs in the interface region. During tensile loading, after the elastic region of the stress-strain curve of the off-axis sample, there is a slope change which reflects the onset and accumulation of distributed matrix microcracking throughout the specimen. These micro cracks introduce a different "phase" inside the materials with different conductivity and permittivity than the surrounding material. At the boundary of these newly introduced micro cracks, interfacial polarization occurs and is reflected in Figs. 9 and 10, in which both the real and imaginary part of the permittivity increases. When an internal surface forms, there is in general a charge accumulation at the interior surface and a global initial rise in the permittivity because of the collective charge accumulation (exterior and interior surface). If the volume of the defects/cracks has a bulk permittivity lower than the outside material system, as the volume of the inclusion increases, the charge accumulation at the exterior surface eventually decreases. The amount of interior surface is increasing

*Corresponding Author

E-mail address: reifsnider@cec.sc.edu 
with volume fraction, but the bulk volume is increasing faster than the surface area, so the global decrease due to the volume effect is ultimately dominant, and the global permittivity eventually falls below the initial undamaged value. Six samples were tested for each condition to check the repeatability of the test data and for all cases test results followed the same trend.

Xing et. al. [27] showed that the loading strain rate effects the mechanical response of composite materials similar to those tested here. We considered continuous glass fiber reinforced epoxy coupon specimens, generally five plies thick, $18 \mathrm{~mm}$ wide, and $75 \mathrm{~mm}$ long in the gage section. We tested the coupons at two different loading rates; one set was tested at $300 \mathrm{~N} / \mathrm{min}(16.67 \mathrm{MPa} / \mathrm{min})$ and another set was tested at $600 \mathrm{~N} / \mathrm{min}(33.33$ $\mathrm{MPa} / \mathrm{min}$ ). Fig 11. illustrates that the dielectric properties of our samples were also rate dependent. With higher strain rates there was a permittivity increase at the onset of damage, suggesting that interfacial damage (such as fiber-matrix debonding) may play a larger role in the damage initiation process than it does at lower strain rates [28].

\subsection{Edge Replication: Observation of Damage Progression}

In order to record specific damage information, especially crack initiation and growth, an edge replication method was utilized which is widely used in quantifying crack density [29]. Edge replicas were acquired by pressing an acetone-softened acetate tape against the sample edge. After hardening, the tape revealed permanent details of the sample edge. Images of those tapes were taken with a Leica ${ }^{\mathrm{TM}}$ optical Microscope. Fig. 12 shows an instance of the initial damage and progressive accumulation of the damage until material failure. In zone-II of Fig. 8, distributed micro cracks occur, which were observed in the picture of the edge replica tape for $1 \mathrm{kN}$ and $1.5 \mathrm{kN}$ load levels. The edge replicas for

*Corresponding Author

E-mail address: reifsnider@cec.sc.edu 
the $2 \mathrm{kN}$ and $2.5 \mathrm{kN}$ loads show bridging of the microcracks, which reflects zone-III in Fig. 8. The highest loading images in Fig 12. show cracks going through the fiber tows which also indicates occurrence of fiber fracture as in region -IV of Fig. 8 where the slope of the dielectric property change is higher.

\section{Conclusion:}

In this paper we have introduced a newly developed in-situ, in-operando dielectric measurement method to measure dielectric property changes with increasing micro-crack density and other damage induced by mechanical loading. It is determined that the size, shape and orientation of microdamage as well as the dielectric character of the defect volume and the interfaces created are all distinguished by the method. Observed changes were not monotonic, and were specific to the dominant damage mode as a function of load level.

In general, the dielectric properties of heterogeneous systems are influenced by various physical factors: electrical and structural interactions between particles, heterogeneity of morphological and electrical properties of the constituent phases, frequency dependence of electrical phase parameters, intra-particle structure, particle shape, size, orientation and morphology. This dependence complicates the determination of the electrical parameters of heterogeneous materials from the observed dielectric relaxation spectra, but also presents us with an opportunity to recover important information not only about the electrical and structural properties of constituents but also about the interactions between constituents, including parent material and damage phases. Further theoretical and experimental investigation is required to fully understand the changes in dielectric spectra associated with specific damage accumulation events in heterogeneous material systems.

*Corresponding Author

E-mail address: reifsnider@cec.sc.edu 
From the results presented in this paper, it can be concluded that analysis of the dielectric data gives us information about the type of material state change throughout the mechanical life of the composite material. It should be emphasized that these changes in the dielectric properties are distinct and measurable changes in material state, and that they are caused by a non-conservative, non-equilibrium material response to the applied fields.

\section{Acknowledgment}

The Authors would like to acknowledge the support to conduct this research by the DOE Energy Frontier Research Center (EFRC) at the University of South Carolina and DARPA, DOE / BES Grant \# DE-SC0001061.

\section{References}

[1]. Reifsnider, K.L., and Case, S. W. (2002), Damage Tolerance and Durability of Material Systems. New York: John Wiley and Sons.

[2]. Gates, T. S. (1991). Rate Dependent Stress-Strain Behavior of Advanced Polymer Matrix Com- posites. NASA TM 104070, NASA Langley Research Center

[3]. Hufner, D. Progressive Failure of Woven Polyer-Based Composites under DynamicLoading: Theory and Analytical Simulation. University of Connecticut, PhD Dissertation (2008).

[4]. Prasse, T., Michel, F., Mook, G., Schulte, K., \& Bauhofer, W. (2001). A comparative investigation of electrical resistance and acoustic emission during cyclic loading of CFRP laminates. Composites science and technology, 61(6), 831-835.

[5]. Rippert, L., Wevers, M., \& Van Huffel, S. (2000). Optical and acoustic damage detection in laminated CFRP composite materials. Composites science and technology, 60(14), 2713-2724.

*Corresponding Author

E-mail address: reifsnider@cec.sc.edu 
[6]. Maslov, K., Kim, R. Y., Kinra, V. K., \& Pagano, N. J. (2000). A new technique for the ultrasonic detection of internal transverse cracks in carbon-fibre/bismaleimide composite laminates. Composites science and technology, 60(12), 2185-2190.

[7]. Steiner, K. V., Eduljee, R. F., Huang, X., \& Gillespie Jr, J. W. (1995). Ultrasonic NDE techniques for the evaluation of matrix cracking in composite laminates. Composites science and technology, 53(2), 193-198.

[8]. Gao, S. L., \& Kim, J. K. (1999). Scanning acoustic microscopy as a tool for quantitative characterization of damage in CFRPs. Composites science and technology, $59(3), 345-354$.

[9]. Vavouliotis, A., Paipetis, A., \& Kostopoulos, V. (2011). On the fatigue life prediction of CFRP laminates using the electrical resistance change method. Composites Science and Technology, 71(5), 630-642.

[10]. Athanasopoulos, N., \& Kostopoulos, V. (2012). Resistive heating of multidirectional and unidirectional dry carbon fiber preforms. Composites Science and Technology, 72(11), $1273-1282$.

[11]. Sevkat, E., Li, J., Liaw, B., \& Delale, F. (2008). A statistical model of electrical resistance of carbon fiber reinforced composites under tensile loading. Composites Science and Technology, 68(10), 2214-2219

[12]. Louis, M., Joshi, S. P., \& Brockmann, W. (2001). An experimental investigation of through-thickness electrical resistivity of CFRP laminates. Composites science and technology, 61(6), 911-919.

[13]. Schueler, R., Joshi, S. P., \& Schulte, K. (2001). Damage detection in CFRP by electrical conductivity mapping. Composites Science and Technology, 61(6), 921-930

*Corresponding Author

E-mail address: reifsnider@cec.sc.edu 
[14]. Xia, Z. H., \& Curtin, W. A. (2008). Damage detection via electrical resistance in CFRP composites under cyclic loading. Composites science and technology,68(12), 25262534.

[15]. Fankhänel, B., Müller, E., Mosler, U., Siegel, W., \& Beier, W. (2001). Electrical properties and damage monitoring of SiC-fibre-reinforced glasses. Composites science and technology, 61(6), 825-830

[16]. Abry, J. C., Choi, Y. K., Chateauminois, A., Dalloz, B., Giraud, G., \& Salvia, M. (2001). In-situ monitoring of damage in CFRP laminates by means of AC and DC measurements. Composites Science and Technology, 61(6), 855-864.

[17]. Böger, L., Wichmann, M. H., Meyer, L. O., \& Schulte, K. (2008). Load and health monitoring in glass fibre reinforced composites with an electrically conductive nanocomposite epoxy matrix. Composites Science and Technology, 68(7), 1886-1894.

[18]. Todoroki, A., Omagari, K., Shimamura, Y., \& Kobayashi, H. (2006). Matrix crack detection of CFRP using electrical resistance change with integrated surface probes. Composites science and technology, 66(11), 1539-1545.

[19]. Vavouliotis, A., Paipetis, A., \& Kostopoulos, V. (2011). On the fatigue life prediction of CFRP laminates using the electrical resistance change method. Composites Science and Technology, 71(5), 630-642.

[20]. Xia, Z., Okabe, T., Park, J. B., Curtin, W. A., \& Takeda, N. (2003). Quantitative damage detection in CFRP composites: coupled mechanical and electrical models. Composites science and technology, 63(10), 1411-1422.

*Corresponding Author

E-mail address: reifsnider@cec.sc.edu 
[21]. Irving, P. E., \& Thiagarajan, C. (1998). Fatigue damage characterization in carbon fibre composite materials using an electrical potential technique. Smart materials and structures, 7(4), 456.

[22]. Agilent Technology Application Note 1217-1(1992). "Basics of measuring the dielectric properties of materials" Agilent, Palo Alto, CA.

[23]. Fazzino, P. D., Reifsnider, K. L., \& Majumdar, P. (2009). Impedance spectroscopy for progressive damage analysis in woven composites. Composites Science and Technology, 69(11), 2008-2014.

[24]. Reifsnider, K. L., Fazzino, P., Majumdar, P. K., \& Xing, L. (2009). Material state changes as a basis for prognosis in aeronautical structures. Aeronautical Journal, 113(1150), 789-798 (Silver Award, 2010, Royal Aeronautical Society).

[25]. Huray, P. G. (2011). Maxwell's equations. John Wiley \& Sons.

[26]. Kremer F., Schonhals A., Broadband Dielectric Spectroscopy, in Springer-Verlag, 2002.

[27]. Xing, L., Reifsnider, K. L., \& Huang, X. (2009). Progressive damage modeling for large deformation loading of composite structures. Composites Science and Technology, 69(6), 780-784.

[28]. Yang., W. and Pelegri, A., (2012). Strain Rate Effect on the Stiffness of Random Fiber Composites due to Matrix Cracking and Interfacial Debonding. Mechanics of Solids, Structures and Fluids, 8, 673-680.

[29]. Masters, J. E., \& Reifsnider, K. L. (1982). An investigation of cumulative damage development in quasi-isotropic graphite/epoxy laminates. ASTM STP, 775, 40-62.

*Corresponding Author

E-mail address: reifsnider@cec.sc.edu 
[30]. Tuncer E., Serdyuk Y. V. and Gubanski S. M. (2002). Dielectric mixtures: electrical properties and modelling. IEEE Trans. Dielectr. Electr. Insul. 9 809-28

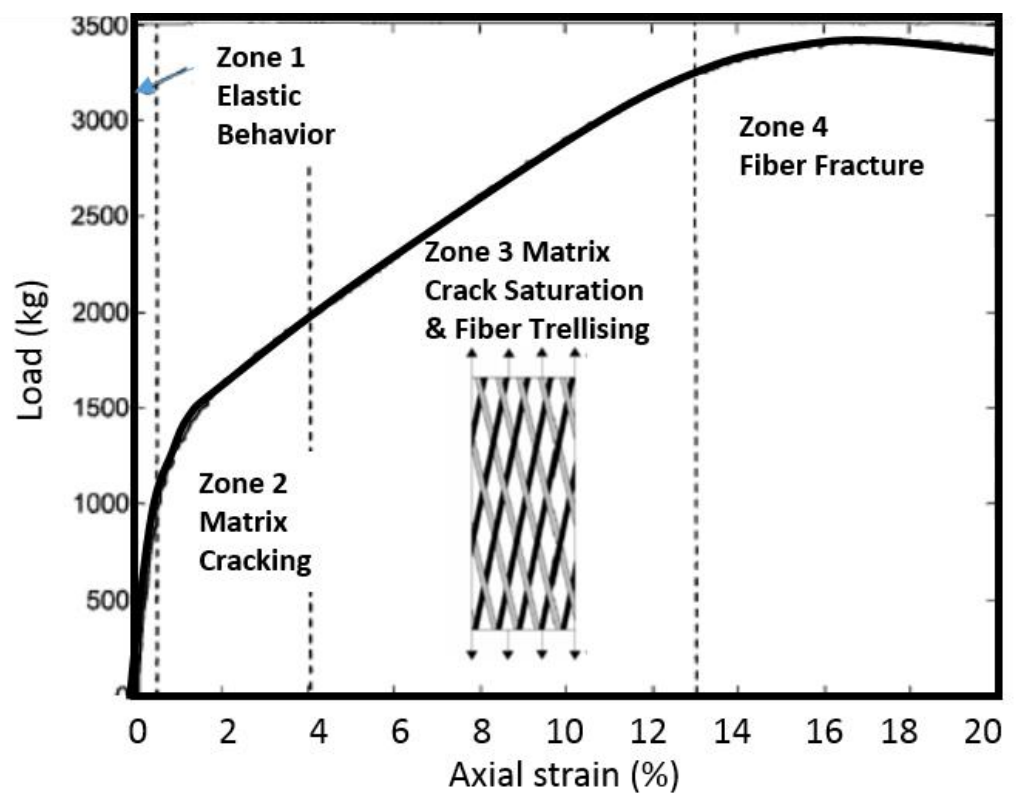

Fig1. Off-axis Response of Woven Composite (45 Degree Tension) [13]

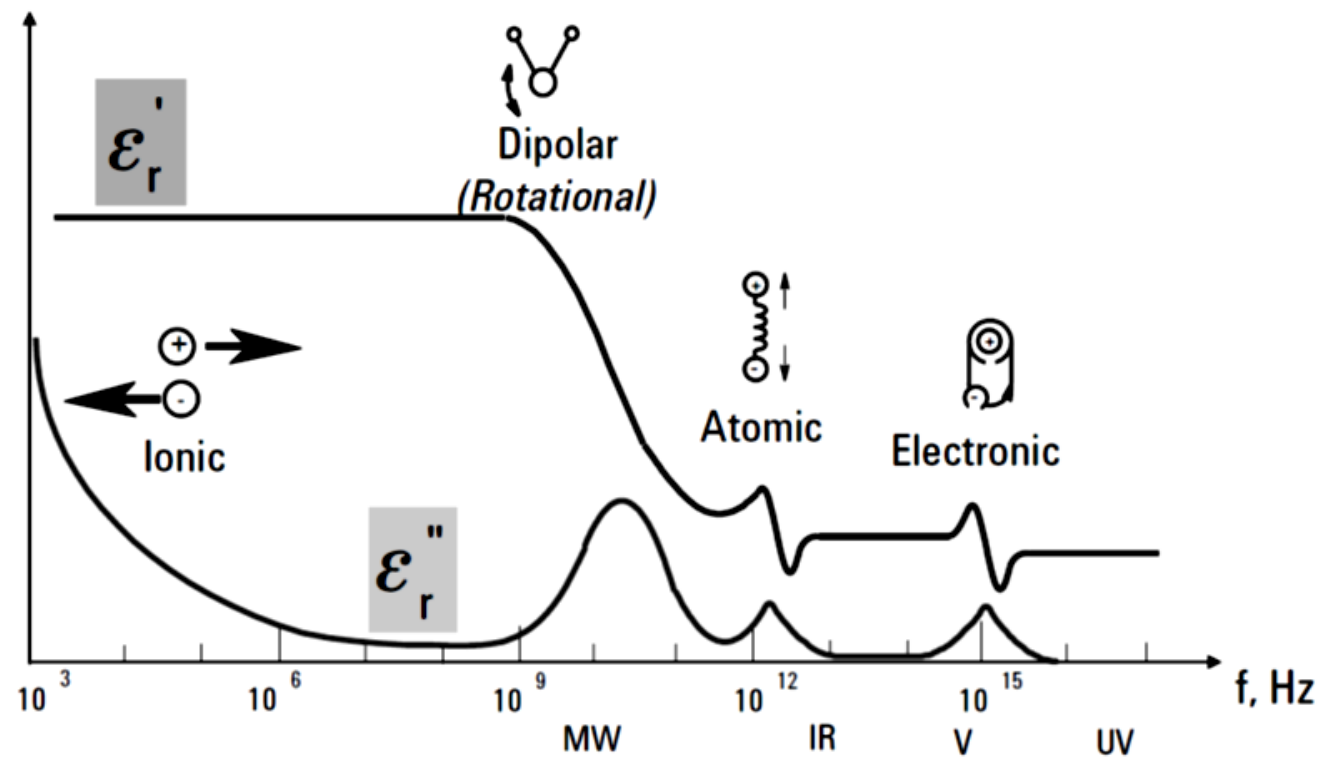

Fig 2. Dielectric responses of material constituents at broad band frequency range [22].

*Corresponding Author

E-mail address: reifsnider@cec.sc.edu 


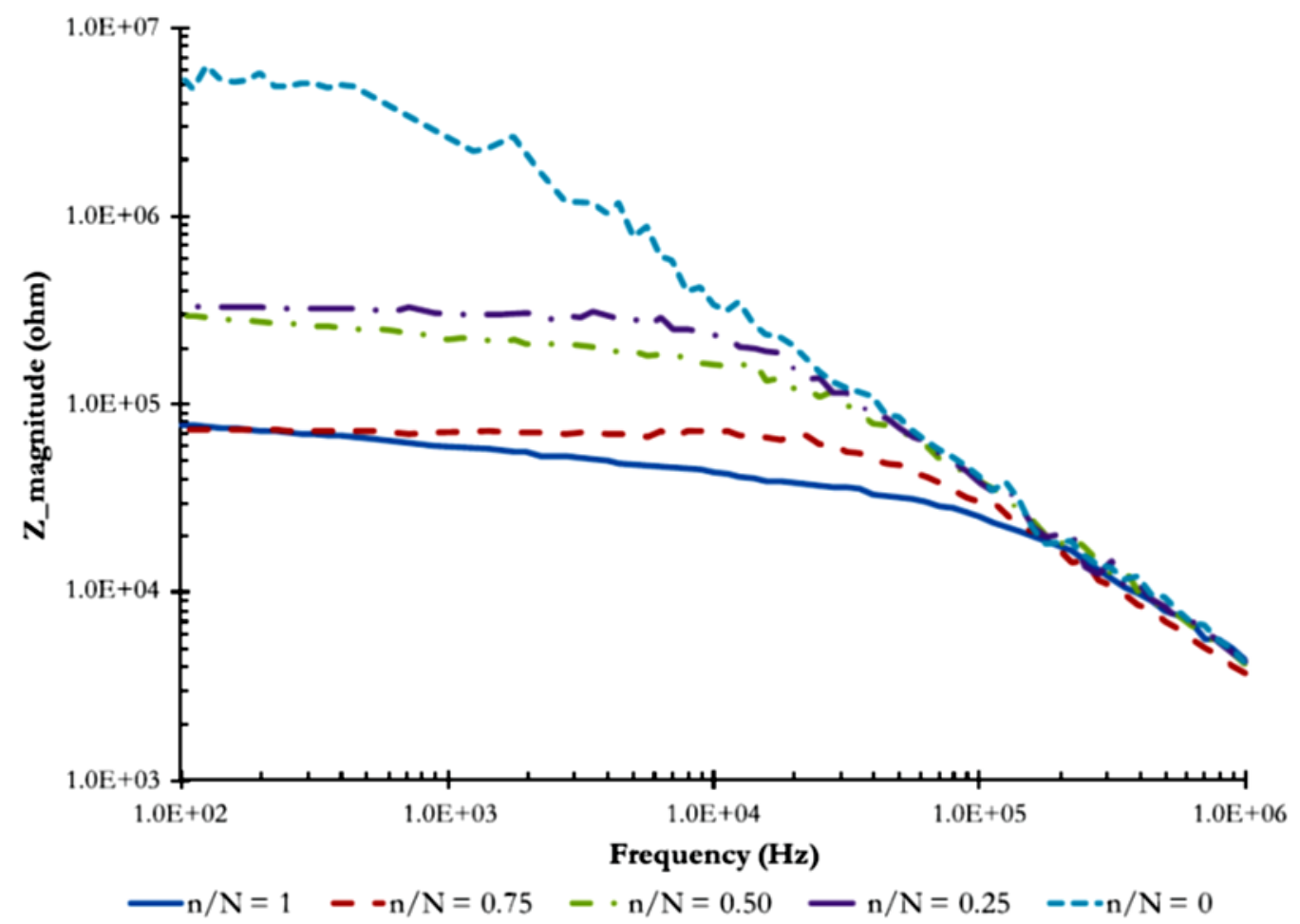

Fig 3. Impedance magnitude versus frequency [23].

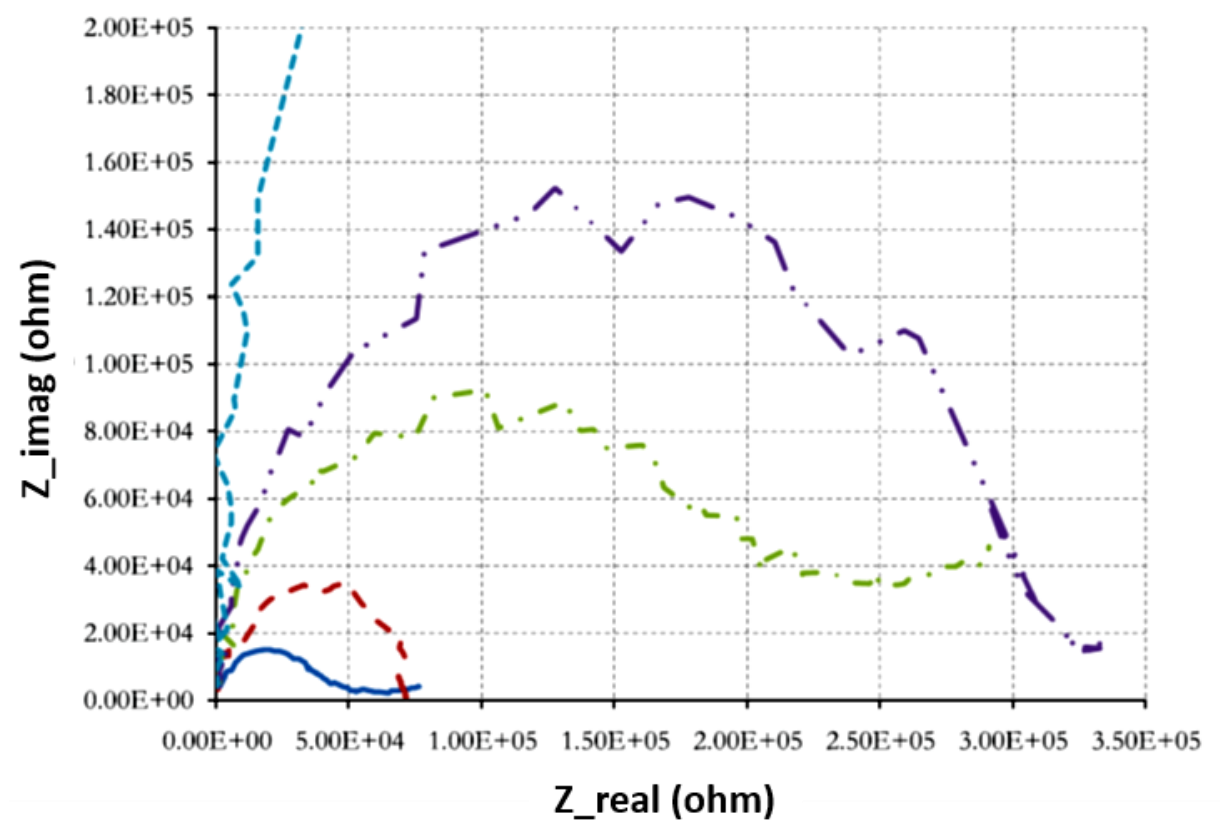

Fig 4. Nyquist plot [23] 


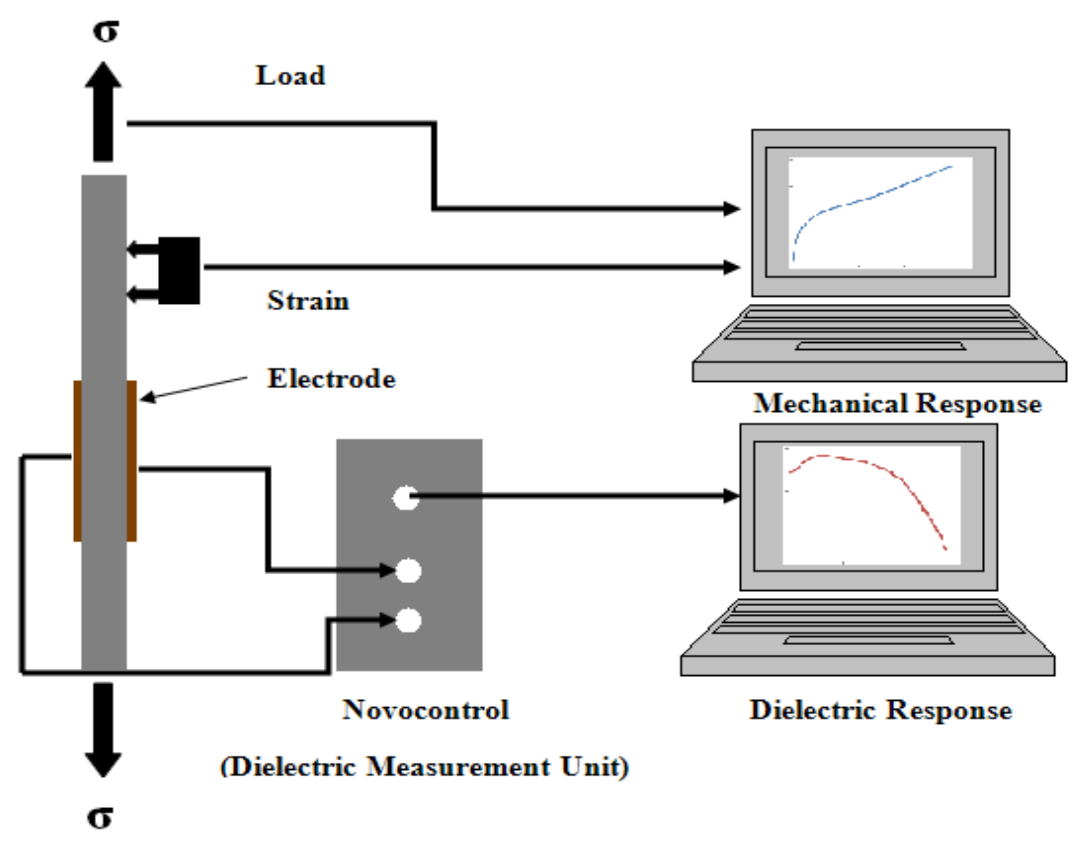

Fig 5. Schematic of the experimental setup

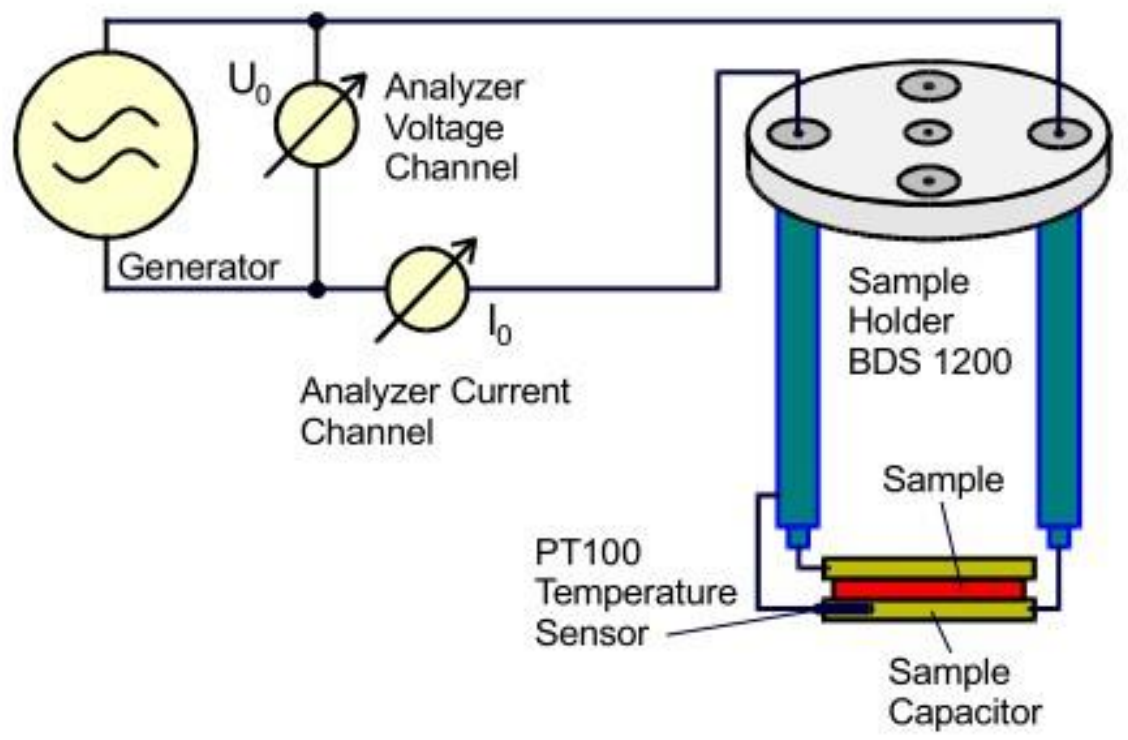

Fig 6. Principle of a dielectric or impedance measurement 


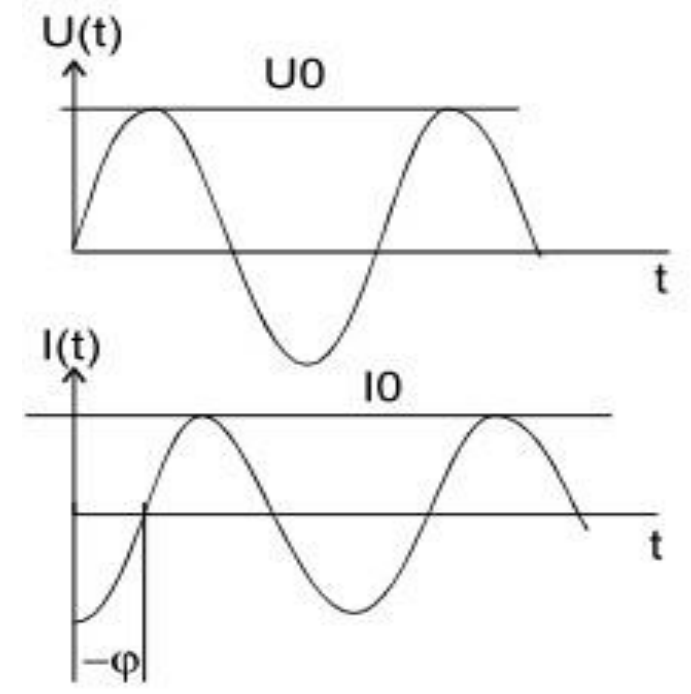

Fig 7. Amplitude and phase relations between voltage and current of a sample capacitor for electric measurements. 


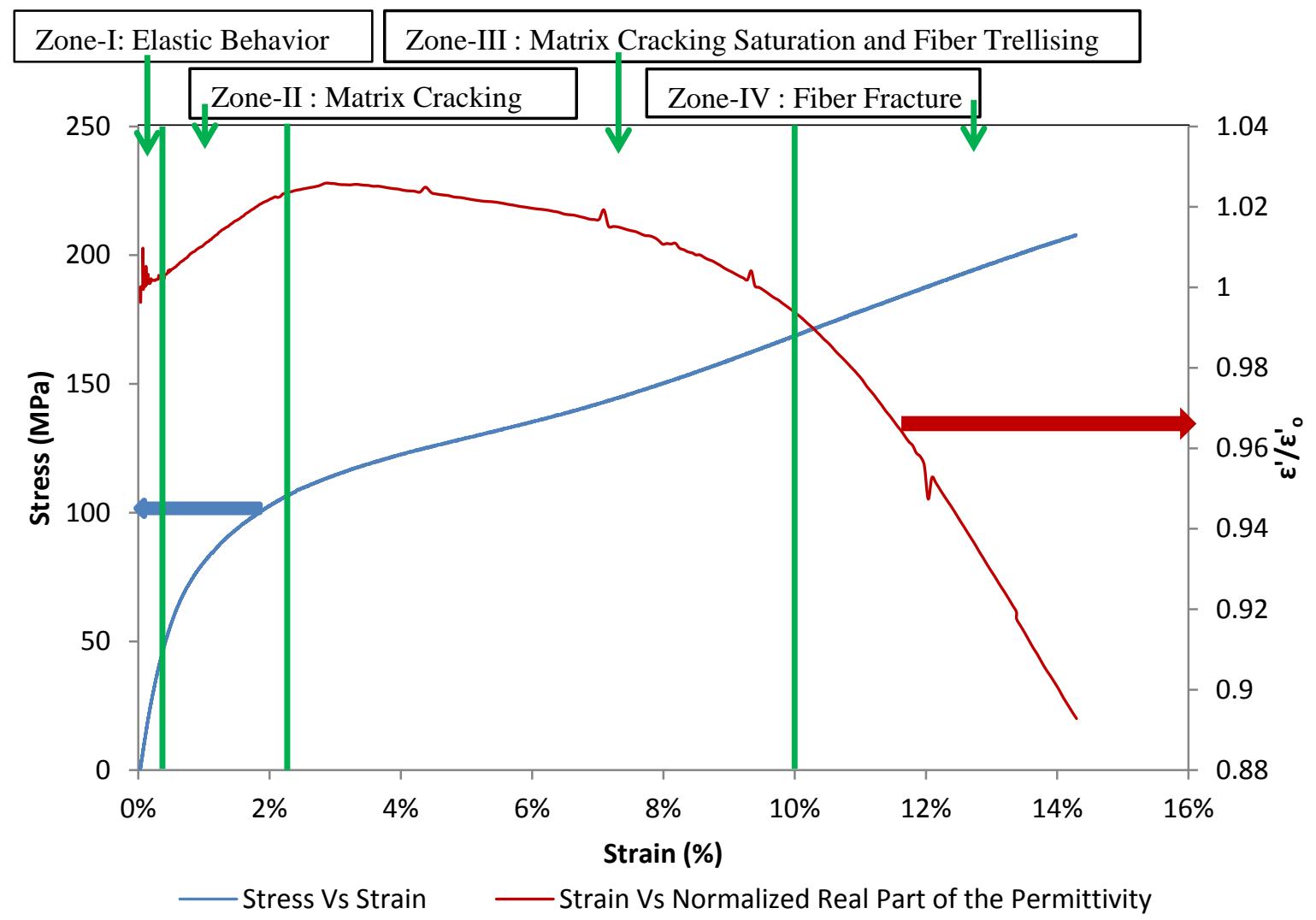

Fig 8. Response of the dielectric property in different zones of damage progression.

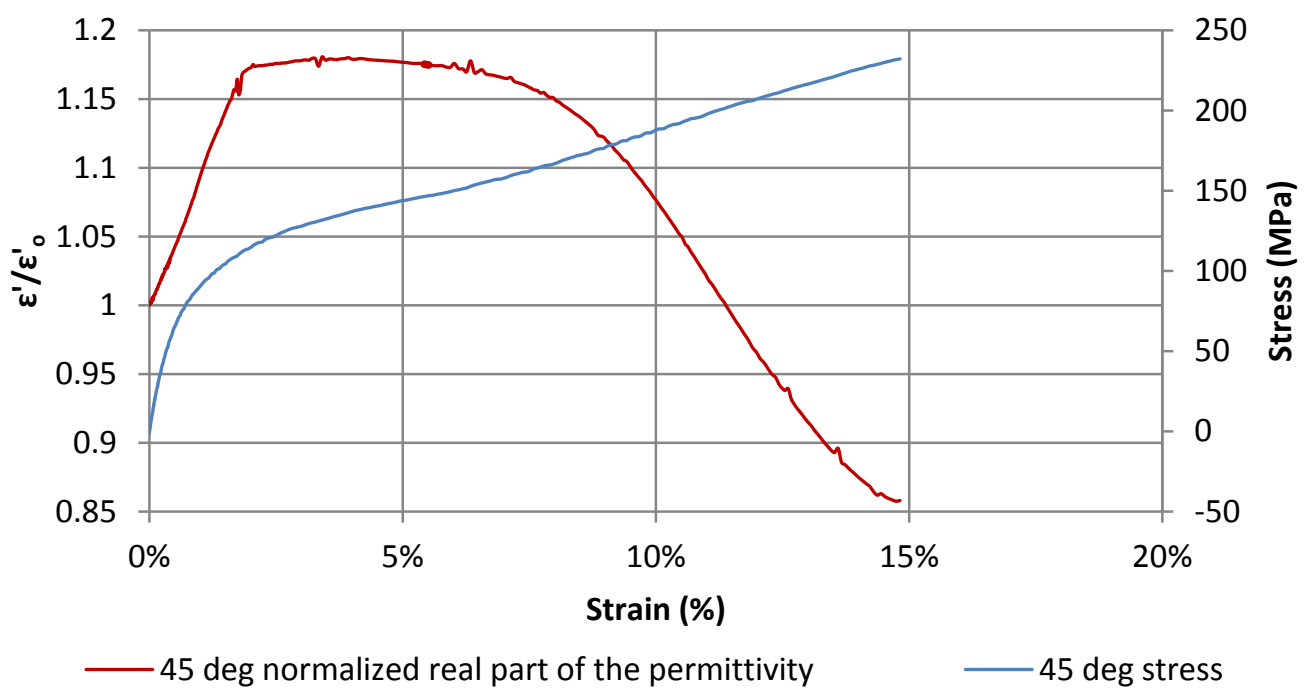

Fig 9. Normalized real part of the permittivity and Stress Vs Strain of an off-axis sample

*Corresponding Author

E-mail address: reifsnider@cec.sc.edu 


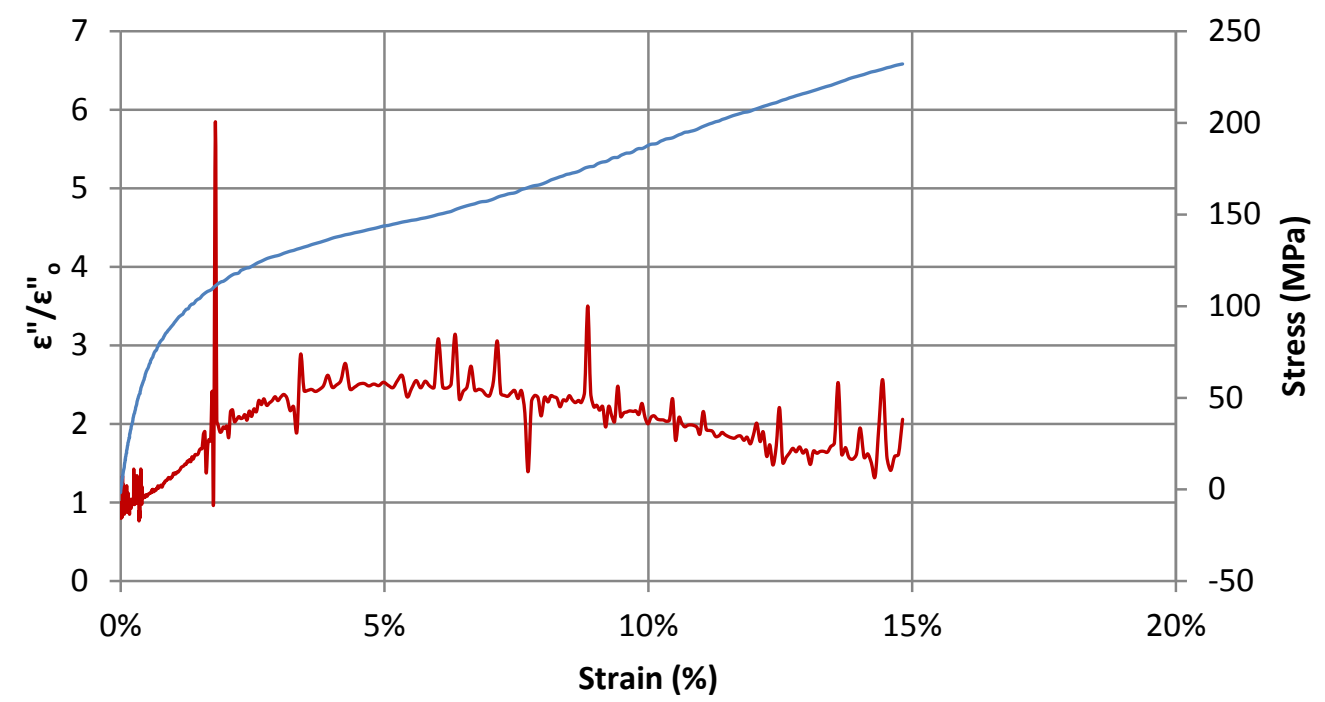

— 45 deg normalized imaginary part of the permittivity $\quad$ _ 45 deg stress

Fig 10. Normalized Imaginary part of the permittivity and Stress Vs Strain

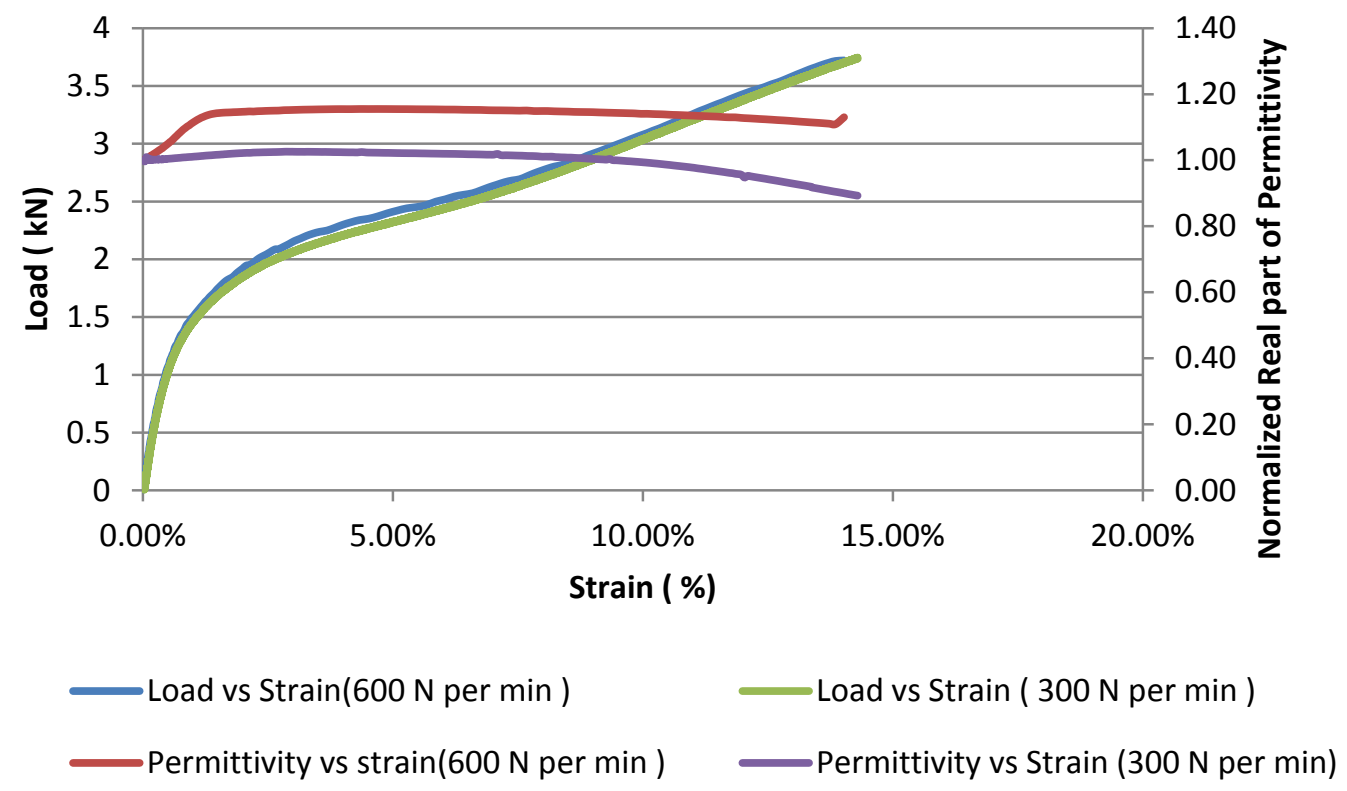

Fig 11. Rate dependency of the dielectric properties. 

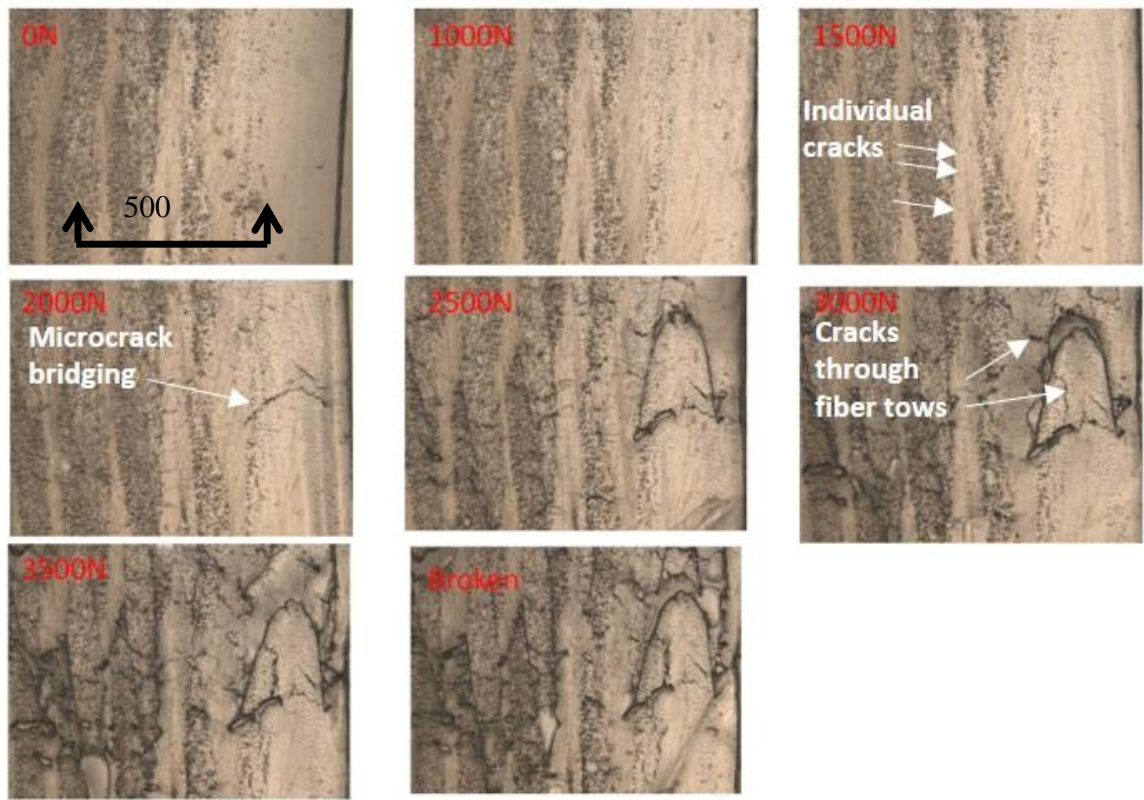

Fig 12. Edge replica at different Load 